\title{
Review of "Perception and production of linguistic and musical rhythm by Korean and English middle school students" by Lydia N. Slobodian
}

\author{
JOHN R. IVERSEN \\ The Neurosciences Institute, San Diego, CA, USA [1]
}

\begin{abstract}
Interest in possible cultural influences on basic rhythm perception and production has been growing, and the paper by Slobodian (2008) fits squarely in this trend, studying rhythm perception and production in a large number English and Korean native speakers. The findings were interpreted in terms of cross-cultural similarity, suggesting that preferences, e.g. for binary meter, are broadly shared across cultures. As is commonly encountered in cross-cultural research, however, there were several difficulties in offering a clear interpretation of the results, such as the large extent of Western music enculturation of the Korean participants. This commentary will review Slobodian's findings, offering an alternative interpretation of one result, suggesting there may be a cultural difference in meter perception. It will also review other relevant research and integrate lessons learned from a recent study of rhythm perception in Korean, American and Japanese listeners. Throughout, it aims to offer suggestions of how to improve the chances of reaching the ultimate goal of understanding what aspect of culture shapes rhythm perception and production, and the mechanisms by which they do so.
\end{abstract}

Submitted 2008 December 1; accepted 2008 December 3.

KEYWORDS: rhythm, meter, language, cross-cultural methodology

IN a creative and ambitious study in this issue (Slobodian, 2008), Korean and English speakers were tested with a battery of rhythm perception and production tasks, covering both speech and musical rhythm, in order to determine if culture plays a role in shaping such behaviors. Several tendencies (toward binary meters and reproducing rhythms using small integer duration ratios) have been found in prior work (and are summarized by Slobodian), but their universality is a matter of debate, for there is evidence that they might depend on culture (Hannon and Trehub, 2005; Sadakata, et al., 2004; Ohgushi, 2006), but also that there might exist predispositions for duple meter (Bergeson and Trehub, 2006). The present work seeks to expand the range of this question to include Korean.

Two culture-dependent areas of experience are typically proposed to account for differences in rhythmic perception and production: language and music. (The alternative, genetic difference, is infrequently proposed.) Slobodian follows this trend, citing studies by Patel, Huron and colleagues, that show the rhythm of a language (as measured by a simple metric) is reflected in the rhythms of composed concert music. This earlier research suggests the existence of links between language and music rhythm, but examines the rather rarefied domain of historical composition in looking for such expression. The search for the substance of such links is generally conducted using behavioral studies in the field or lab. The current research thus falls within two traditions: the comparison of language and musical rhythm using simple metrics, and the drive to test if results in music cognition hold cross-culturally, as a way to understand to what extent they may be universal versus learned (e.g. Grabe and Low, 2002; Patel \& Daniele, 2003; Drake and Bertrand, 2001).

\section{COMMENTARY ON RESULTS}

Two main questions were addressed experimentally: 1) How do the speech rhythms of Korean and English differ and 2) are the same tendencies in rhythm perception and production seen in Western listeners also seen in Korean listeners? In addition, a questionnaire about music experience was administered. Participants were middle school students from Korea, America or England (the latter two were pooled as the English-speaking group).

Within these questions, there were several underlying aims of the work: First, to see if there is a connection within language between language rhythms and musical rhythm perception/production; Second, to 
provide some basic empirical characterization of Korean speech rhythms, in particularly testing if it has less durational variability than English; Third, to test if the rhythmic tendencies of mainly Western, European listeners are shared by listeners from a non-Western culture; Fourth, to ask if aspects of Korean music (e.g. lack of anacrusis) affect beat perception differently.

\section{Speech rhythms}

There is disagreement about the rhythmic features of Korean speech, making it difficult for the author to formulate clear predictions on how musical rhythm production and perception might be affected by Korean language. Slobodian understands this point, as part of her study directly measured language rhythms of Korean and English speakers. This is an excellent idea, especially when done within the same group being tested with the other tasks. All participants read aloud sentences in Korean, English and Emakuah (a syllabically similar language unknown to all). The Normalized Pairwise Variability Index (nPVI) was used to measure the variability of several types of successive intervals in speech (Grabe and Low, 2002). Interestingly, no differences were found across all the varied dimensions of possible comparison, within and across language groups. The consequence for the study is that there is no rhythmic difference in language that might predict differences in rhythm perception or performance. However, there is reason to question the generality of this finding of similarity in language rhythms.

A methodological issue explained by the author is that the variability between individual sentences within the same language was so great as to mask effects of the language of the speaker. It could be added that the present test was too broad in its aims for the small number of sentences used, and was not so well suited for the purposes at hand, which is to understand the rhythmic biases that might differentiate Korean and English. Ultimately, to answer the many interesting questions inherent in the present design, a more extensive study is necessary. There are standard, longer, sets of sentences that have been used in previous studies (e.g. Mok and Lee, 2008) that would be useful to use in future work to be able to compare results across studies.

In this regard, it would also have been helpful if numerical results had been presented, to enable comparison of the measured values with the growing collection of other studies that have made such measurements. It appears from recent work (Mok and Lee, 2008) that examination of a wider range of measurements is necessary to describe Korean: it resembles syllable-timed languages when examining the PVI of syllables, but, strikingly, has an even larger vocalic nPVI than even English, a stress-timed language. Clearly, more work is needed to characterize Korean. It is not clear, even, that $\mathrm{nPVI}$ and measures related to linguistic classification are the most relevant. Perhaps most importantly, some hypothesis of how language rhythm might be expected to influence musical rhythm perception and production needs to be articulated to guide such measurements!

\section{Musical rhythm perception and production}

The heart of Slobodian's study is a set of three tests of rhythm production and perception. The first two assessed accuracy in performing duration ratios, while the last tested beat perception and production. The aims of this section were to replicate earlier findings in Western listeners on related tasks, and extend them to a new culture group.

Tasks 2 and 3 tested the accuracy of reproducing short-long rhythm elements with a ratio of 2:1 or 3:1 in durations. Task 2 featured repeating short-long rhythms, while task 3 placed these ratios within short rhythms featuring 2:1, 3:1 or mixed ratios. Participants reproduced the stimuli by clapping. Results for the Korean and English-speaking participants was largely similar, and replicated earlier findings of the 'compression' of more extreme ratios such as 3:1 towards 2:1, although it is clear that the 2:1 ideal was often not accurately reproduced. Koreans generally had slightly (but significantly) larger ratios (more swing) in reproducing the 2:1 intervals, which were typically underestimated by English-speaking participants.

Task 4 asked listeners to clap along with the beat of various rhythms. This is potentially the most interesting task, given differences between metrical organization in Western and traditional Korean music, and the wide range of possible metrical interpretations participants can express. It was also examined if the lack of anacrusis in traditional Korean music might be reflected in this meters selected by participants. This task was apparently difficult to explain, and was extended somewhat in a second version. The beats chosen by listeners showed a large amount of variety, with binary or compound meters, with or without anacrusis, being chosen by different listeners for the same rhythm. Such variety tends to be the norm in beat finding studies (e.g. Iversen, et al, 2008). Several measures were made to characterize metrical interpretations: competence, production of binary meters, and presence of anacrusis in a chosen metrical interpretation. The most striking finding was that Korean participants had much lower competence than English speakers. It is suggested that competence was poor 
because the task was not clearly explained, but it is puzzling why it should be nearly half that of English participants.

Along most other measures, the two groups tended to be similar. For example Korean listeners were just as likely to interpret fast rhythms as anacrustic as their English counterparts, so the absence of anacrusis in Korean music seemed not to influence the participants' beat finding.

The general conclusion drawn by Slobodian from the musical rhythm tasks is that Korean participants behave similarly to English participants, suggesting that the preference for binary meter and small interval ratios, if not universal, is at least also observed in a non-western culture, though 'to a lesser extent.'

There may, however, be another way of reading the results on the tendency to hear binary meters (Figure 12 in Slobodian, 2008) that doesn't view the Korean results as simply an attenuated version of English results. Specifically, Korean listeners more consistently interpreted rhythms containing 2:1 ratios in compound meter, but English listeners switched to binary interpretations at slow tempi, even though such interpretation required syncopation. That is, Korean listeners may have been more aware of the 'threeness' of such rhythms, or have been more comfortable grouping in threes. Though testing such a suggestion would need further study, such a tendency would seem to fit at least with the prevalence of ternary structures in Korean traditional music, to which all Koreans had been exposed.

\section{Methodological issues}

Slobodian clearly points out a variety of methodological issues that bear on the interpretation of the results. Some of these are inherent in the type of in-school field experiments that were used (e.g. background noise), but these seem to have been handled well. Others are more problematic: short time allotted to the experiments and difficulty in explaining the experiment. Regarding time, which was limited to about seven minutes per participant, it's impressive the range of experiments Slobodian was able to perform. A large number of participants were used to counter the inherent noisiness of such a short experiment. It's to be hoped that a second iteration of this of study could focus on fewer participants, for a longer period of time. As for the difficulties in communication, they seemed to have complicated the interpretation of the current results, and must be resolved.

\section{RELATED RESEARCH}

Several recent studies not noted by Slobodian directly bear on the current results, and are worth examining. Azechi (2005) found that English and Japanese speaking children diverged in their singing of dotted rhythms as they developed, with the Japanese children's singing more in line with the lower interval variation of Japanese language. This finding parallels that by Ohgushi (2006) on piano performance of dotted rhythms, which finding smaller performed ratios in Japanese student pianists. Of interest for the current study, Ohgushi also studied Korean student pianists, and found that their performance of dotted rhythms was closer to that of Europeans than Japanese. This result is generally consistent with the present work.

A different measure of rhythm perception, the rhythmic grouping of sequential elements in patterns, has recently been studied cross-culturally (Iversen, Patel and Ohgushi, 2008). It was found that that grouping of sequences of alternating duration tones is not universal, but differs in English and Japanese listeners. This difference in preference was hypothesized to arise from the different syntax and word rhythms of the two languages. Observation of rhythmic differences in language that match non-linguistic perceptual differences was coupled with a developmental hypothesis concerning the learning of phrase and word segmentation to suggest that learning the rhythms of language has deep effects on general rhythm perception. This view has recently received support from a study on English and Japanese learning infants, showing that they develop grouping preferences that match adults of their culture at 7-8 months, about the time that word segmentation emerges (Yoshida, et al, 2008). 
a

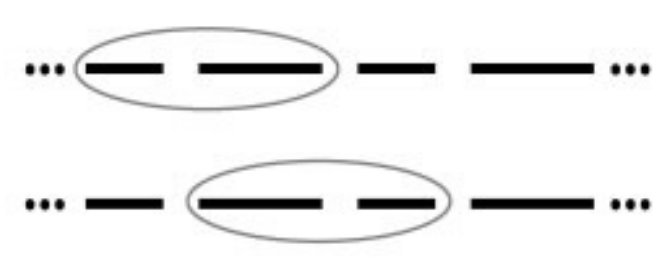

b

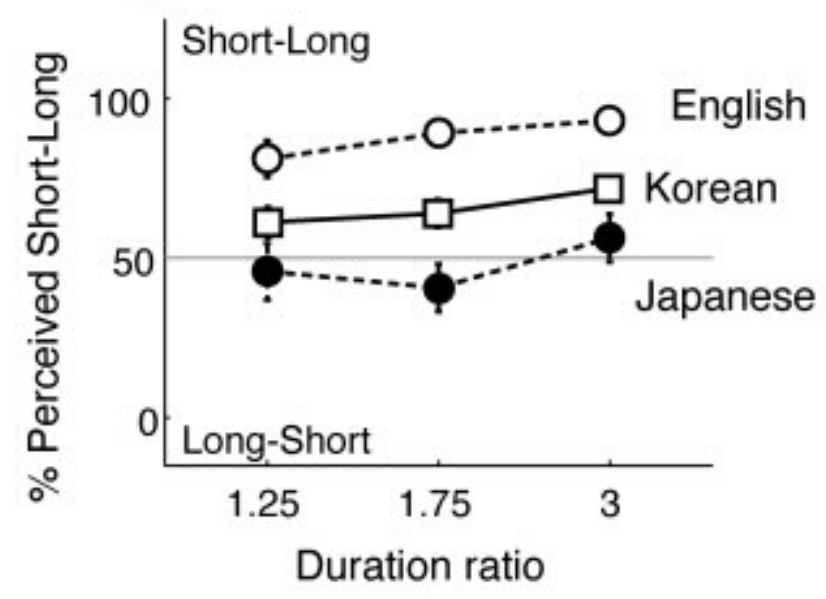

Fig. 1. (a) Schematic of stimulus sequence of tones alternating in duration. Listeners heard duration rhythms with several different duration ratios between short and long (1:1.25, 1:1.75 and 1:3). For each stimulus, listeners reported if they heard it grouped as short-long, or long-short (indicated by ovals). (b) Grouping preferences. Both English (dashed line, solid circles) and Korean (solid line) speakers have stronger preference for short-long grouping, though the Korean preference is not as strong. Language differences are highly significant (ANOVA, $\mathrm{F}(2,431)=39, \mathrm{p}<0.0001)$. Collapsing across duration ratios, means for each language are significantly different when considered in post-hoc pairwise tests between languages: English, 87.2\%; Korean, 65.6\%; Japanese, 43.8\% (all p $<0.003$; Wilcoxon rank-sum). English and Japanese data are from Iversen, et al. (2008). Korean data is a sample of 85 female university students at Ewha Women's University in Seoul.

Recently, this work has been extended to Korean listeners, with results summarized in Figure 1. Listeners heard patterns of alternating short and long tones and answered if they heard them grouped short-long or longshort (Fig. 1 a). Korean listeners (solid line) preferred short-long groupings, like the English listeners, but not as strongly (Fig. $1 \mathrm{~b}$; see caption for experimental details). Interestingly, the syntactic hypothesis advanced by Iversen, et al. (2008), taken alone, would have predicted the Korean data to be more like the Japanese results, as both are postpositional. It is clear that arguments from linguistic theory and rhythmic classifications only go so far, and actual measurements are needed to resolve this. In the case of grouping perception, the hypothesis suggests what aspects of language rhythm to quantify, namely of relative syllable durations in short words and phrases.

Several general lessons were learned in the course of the grouping work that could also apply to the current paper. First, group-mean data provided an inaccurate picture of what individual listeners are hearing. Japanese listeners did not resemble the mean (i.e. in having no preference) but instead had strong, heterogeneous preferences. Second, interpretation of the heterogeneity in results would have been aided by having information about each individual's degree English proficiency to rule out (or in) the same kinds of issues of cross cultural experience raised by the present study. Finally, we found it was necessary to have all instruction and experimental materials delivered only in participants' native language, out of a concern for the possibility of context effects. 


\section{COMMENTARY ON INTERPRETATION}

Ideally, one would aim for a definitive answer regarding the universality vs. culture-dependence of rhythm perception and production, as well as evidence regarding what aspects of culture shape behavior. In practice this is often difficult. Slobodian took several laudable steps to enable such interpretation, including measurements of language rhythms, and a questionnaire about music experience, but is forthright in noting the limits of interpretation of the results.

Despite this obvious care taken in the types of data collected, it was disappointing not to see more specific predictions advanced based on known differences between Korean and English language and music. The author may have missed an opportunity to more fully educate the reader regarding Korean language and music, while also developing and articulating specific predictions, which are nonetheless implicit in this work. In the speech study, one assumes there might have been an underlying hypothesis that if there is less durational variability in Korean language then Korean participants might express less durational variability in their rhythmic performance (as has been suggested by Ohgushi, 2006). Regarding music, it is a commonly encountered (though surely simplified) idea that Korean music is often organized into ternary groups. It would have been helpful to have specific hypotheses articulated ahead of time: e.g. Korean listeners will be more likely to hear ternary meters. This does seem to have been the case in the present data.

As it turned out, the attempt to ground the rhythm perception and production results in language was difficult, given this study's observed similarities between Korean and English. However, for reasons discussed already, it is clear that a full characterization of Korean language rhythm is not yet at hand. Suggestions were made above to further this characterization. However, even in the presence of language differences, the degree of English language competence among Koreans would need to be more fully addressed in future studies.

In terms of musical experience, the main finding of the questionnaire was the large degree to which Western musical traditions are in the forefront of the Korean participants' daily experiences. This observation is crucial to have, for while it may limit the ability to interpret the results in terms of cultural differences, it gives concrete reason for this limitation.

Issues of multi-culturalism encountered in this study are naturally shared by many domains of inquiry. (e.g. Duh and Chen, 2007). One way to deal with this is to pick cultures with a clear difference between them in language or music rhythms, yet little overlap in the relevant dimensions of experiences of members of the cultures. Studies of tonal vs. non-tonal languages (e.g. Bent, et al, 2006) and Western vs. Eastern European meters (e.g. Hannon and Trehub, 2005) have successfully taken this route.

More generally, however, overlap between cultures will be present, as in the current study. It is suggested that this makes the identification of the effect of varying experiential factors within a culture all the more important. Thus, a way forward for the present line of research would be to more tightly integrate responses to the music experience questionnaire with the analysis of rhythm perception and production, taking differences in type and duration of musical performance experience into account both across and within groups in order to provide greater insight into the forces shaping participants rhythmic behavior. For example, a natural question that was not discussed in the interpretation of the rhythm production results is the degree and type of musical performance experience of the participants. One would expect musical training to have a large effect on such tasks as repeating rhythms and clapping a beat, and training in Korean traditional music to magnify whatever differences are apparent in the group data. The questionnaire appears to contain the requisite information to accomplish this: A large number of musicians were identified, some studying Western traditions, others Korean. Could it then have been possible to examine the influence of factors measured in the questionnaire, such as music listening habits, and crucially, the level of musical experience in Western or Korean? This would entail having full details on music (and language) experience for each of the participants in the perception/production studies. If this is so, then the data may be on hand to attempt such an analysis, especially given the large number of Korean participants.

More generally, a number of basic issues at the foundations of cross-cultural work will need to be addressed in the future. Perhaps foremost is the extent to which any cultural differences, if found, are relatively permanent features of an individual's perceptual tendencies (as has been suggested for word segmentation strategies, e.g. Cutler, 2000), or if they are more labile, and dependent on recent context. Such a consideration is especially important in accounting for bilingual participants, such as the Korean students in the present study, and can be addressed using studies of bilinguals and longitudinal designs. Second, as in past work (e.g. Hannon and Trehub, 2005) important experimental allies moving forwards will be provided by developmental studies, in the establishment of mechanisms, as well as by music psychology, musicology and linguistics in providing the necessary background for proposing hypotheses about the most relevant paths of connection between domains. 


\section{NOTES}

[1] Correspondence regarding this manuscript should be addressed to:

John Iversen

The Neurosciences Institute

10640 John J Hopkins Dr

San Diego, CA 92121, USA

iversen@nsi.edu

\section{REFERENCES}

Azechi, N. (2005). Dotted rhythm in Japanese and in English: Towards an understanding of the influence of mother tongue on young children's musical behavior. In The Proceedings of the First European Conference on Developmental Psychology of Music, November, 2005, Jyväskylä, Finland. p. 256-259.

Bent T., Bradlow A. R., and Wright B. A. (2006). The influence of linguistic experience on pitch perception in speech and non-speech sounds. Journal of Experimental Psychology: Human Perception and Performance, 32, 97-103.

Bergeson, T. R. and Trehub, S. E. (2006). Infants' perception of rhythmic patterns. Music Perception, 23, 345-360.

Cutler, A. (2000). Listening to a second language through the ears of a first. Interpreting, 5, 1-23.

Deutsch, D. (1991). The tritone paradox: An influence of language on music perception. Music Perception, 8 , 335-347.

Drake, C. and Bertrand, D. (2001). The quest for universals in temporal processing in music. Annals of the New York Academy of Science, 930, 17-27.

Grabe, E. and Low, E. L. (2002). Durational variability in speech and the rhythm class hypothesis. In C. Gussenhoven and N. Warner (Eds.), Laboratory Phonology 7 (pp. 515-546), Berlin: Mouton de Gruyter.

Hannon, E. E. and Trehub, S. E. (2005). Metrical categories in infancy and adulthood. Psychological Science, $16,48-55$.

Iversen, J. R., Patel A. D., and Ohgushi, K. (2008). Perception of rhythmic grouping depends on auditory experience. Journal of the Acoustical Society of America, 124, 2263-2271.

Iversen, J. R. and Patel, A. D. (2008). The Beat Alignment Test (BAT): Surveying beat processing abilities in the general population. In K. Miyazaki et al. (Eds.), Proceedings of the 10th International Conference on Music Perception \& Cognition (ICMPC10), August 2008, Sapporo, Japan, Adelaide: Causal Productions.

Mok, P. and Lee, S.I. (2008). Korean speech rhythm using rhythmic measures. The 18th International Congress of Linguists (CIL18). Seoul, Korea.

Ohgushi, K. (2006). An analysis of timing microstructure in Mozart's piano sonata K.331.

Journal of the Acoustical Society of America, 120, 3204.

Patel, A. D. and Daniele, J. R. (2003). An empirical comparison of rhythm in language and music. Cognition, $87, \mathrm{~B} 35-\mathrm{B} 45$.

Sadakata, M., Ohgushi, K., and Desain, P. (2004). A cross-cultural comparison study of the production of simple rhythmic patterns. Psychology of Music, 32, 389-403. 
Slobodian, L. N. (2008). Perception and production of linguistic and musical rhythm by Korean and English middle school students. Empirical Musicology Review, Vol. 3, No. 4, 187-204.

Yoshida, K. A., Iversen, J. R., Patel, A. D., and Werker, J. F. (2008). Development of Abstract Grammatical Representation. XVIth Biennial International Conference on Infant Studies Vancouver, British Columbia, Canada. 